\section{Special-purpose Slide Rules as Exemplified by a Rule for Maintaining a Constant Exposure in Successive Autoradiographs with lodine-|3|}

Whenever a particular type of calculation is to be repeated frequently, the use of slide rules, nomograms or graphs eliminates a major part of the time required. This is frequently the case in problems involving isotopes of short half-life. Because radioactive decay is exponential, the conventional mechanical calculator is of little help. An ordinary slide rule is far from ideal, and its use requires familiarity with the mathematics of exponential decay. Therefore a simple special purposo rulo has been devised to solve a specific problem. The utility of this rule should make it of general interest and may encourage the development of other rules for special purposes.

Iodine-131 (half-lifo, 8.14 days) has been used in this laboratory for examining the sealing effectiveness of dental
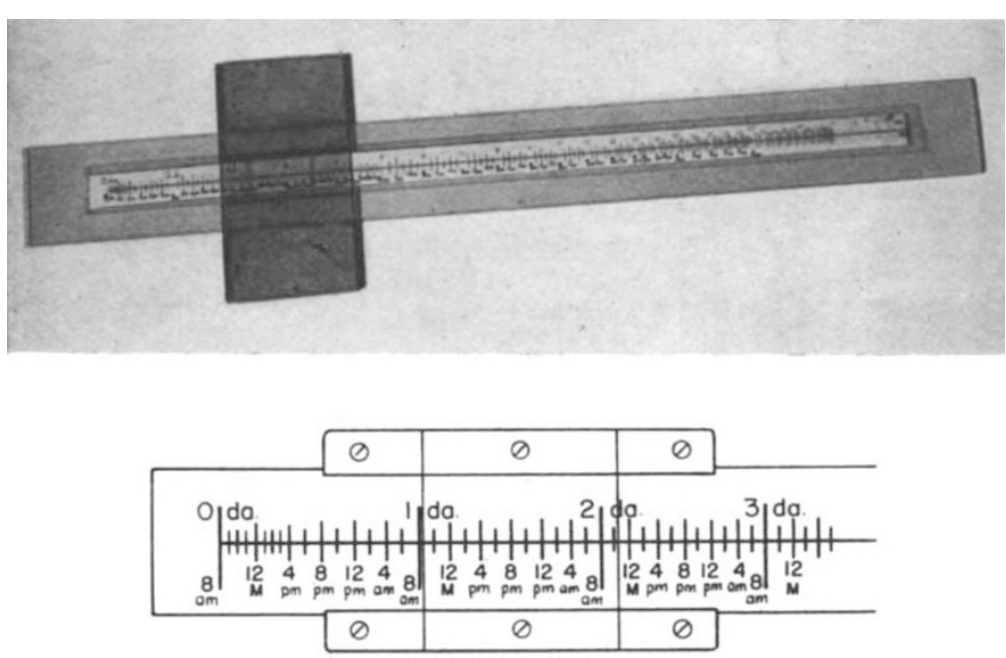

Fig. 1, Plastic slide rule for detcrmining iodine-131 autoradiograph exposure schedule when the initial exposure period is $24 \mathrm{~h}$. Above, the slide rule; line drawing showing detail of portion of scale. Cursor designates an exposuro period from $8 \mathrm{a} . \mathrm{m}$. of day 1 (second day) to $10 \mathrm{a} . \mathrm{m}$. of day 2

fillings. Repeat autoradiographs of soctions are takon following the immersion of the filled teeth in labelled iodide solution. An exposure time of $24 \mathrm{~h}$ was optimal when the tracer solution activity was $250 \mu \mathrm{c}$./ml. (For a graphical solution of exposure when activity of specimen is known see Wainwright, W. W., Anderson, E. C., Hammer, F. C., and Lehman, C. A., Nucleonics, 12, 19; 1954.) Aftor the first exposure, a longer exposure is required to produce the sume density because of radioactive decay. A simple menns of determining successive exposure periods was required. Each exposure should start and end during the hours of the work week; thus use of the semilogarithmic graph of the decay curve was not the best solution.

The exposure of an autoradiograph botween any two times $t_{n}$ and $t_{n+1}, E_{n}^{n+1}$, is proportional to the dissipation of radioactivity during that time, that is :

$$
E_{n}^{n+1}=k\left(a_{n}-a_{n+1}\right)
$$

Letting $a_{0}$ denoto tho initial activity and $\lambda$ the decay constant, the equation for radioactive decay, $-\mathrm{d} a / \mathrm{d} t=\lambda a$, can be written in the integrated form:

$$
a_{n}=a_{0} e^{-\lambda t_{n}}
$$

Equations (1) and (2) can be used to determine the exposure as a function of initial activity and time. Thus, for a standard exposure interval between $t_{0}$ and $t_{s}$ :

$$
E_{0}^{s}=k\left(a_{0}-a_{s}\right)=k a_{0}\left(e^{-\lambda t_{0}}-e^{-2 t_{s}}\right)
$$

If time is mensured from $t_{0}$, this becomes

$$
E_{0}^{s}=K a_{0}\left(1-e^{\left.-\lambda t_{s}\right)}\right.
$$

For any other interval $t_{2}-t_{1}$,

$$
E_{1}^{2}=k a_{0}\left(e^{-\lambda t_{1}}-e^{-\lambda t_{3}}\right)
$$

Equivalent exposure times are obtained where $E_{1}^{2}=E_{0}^{s}$, so that $t_{1}$ and $t_{2}$ must satisfy the relation: $e^{-\lambda t_{1}}-e^{-\lambda t_{2}}=$ $1-e^{-\lambda t} s$. A scale of time graduated according to the function $e^{-\lambda t}$ can therefore be used to ostablish a uniform exposure time.

The rule now in use (Fig. 1) consists of a single timoscale, graduated in this manner, with time in hours and days and a cursor carrying two indices (parallel linos or arrows) the spacing of which is equivalent to the standard exposure $\left(t=0\right.$ to $\left.t=t_{s}\right), 24 \mathrm{~h}$ for our purposes. (To relate the exposure time to the work week the timo scalo runs from 8 a.m. of day 0 in clock-time units: for more general purposes a scale in days and hours starting with $0 \mathrm{~h}$ would be used.) When the cursor is moved so that the left index coincides with the time for starting a new exposure, the time to remove the film will be shown opposite the right index. To determine the spacings on the rule a suitable table of the function $e^{-x}$ is used if available; once $\lambda t$ has been calculated for each value of $t, e^{-\lambda t}$ is read directly from the table. Otherwise, advantage is taken of the fact that $e^{-\lambda t}$ is identical to $10^{-l t}$, where $l=\left(\log _{10} 2\right) / t_{1 / 2}=0.30103 / t_{1 / 2}$. Sinee $\lambda=\log _{e}$ $2 / t_{1 / 2}, l=0.43429 \lambda$. (Tho values of $\lambda$ and $l$ depend on the unit of time. When in days, $\lambda$ and $l$ are 0.08514 and 0.03698 , respectively; when in hours, the values are 0.003548 and 0.001541$.$) \quad A table of antilogarithms, or$ logarithms, can be used to determine 10-lt, tho antilogarithm of $-l t$. The span of the scale, from $t=0$ to $t=\infty$, is $0-1$, or -1 . Therefore the spacings are determinod by subtracting the values of $e^{-\lambda t}$ (or $10^{\text {lt }}$ ) from unity and multiplying by the desired scale length. A table of the function $\left(1-e^{-\lambda t}\right)$ for iodine-131 will be sent on request.

For the rule shown in Fig. 1, tho graduations were inked on millimetre graph paper, transferred to tracing paper, and bonded to a rule of plastic. A clear plastic eursor was fabricated with the 2 index lines inseribed.

The rule as described is for an isotope of 8.14-day halflife. Modifications can be introduced to suit difforent objectives. Replacing this rule with one of broader scope to determine exposure for isotopes of any required half-life increases the comploxity seriously, so that the more specialized rule is usually to be preferred. For the solution of a frequently recurring problom in any field where mechanical calculators are unsuitable or unavailable, the time required to devise a special purpose slide rule is soon repaid.

I thank Barbara Gneier, Timothy Dodge and his staff in the Medical Illustration Service, and John Doemer for assistance in the development of tho rule in Fig. 1.

\section{ELLERY C. Stowell}

Dental Research Laboratory, Veterans Administration Hospital, Long Beach, California,

and Department of Biochemistry,

University of Southern California School of Medicine, Los Angeles. 